\title{
Antigen-specific responses assessment for the evaluation of Bordetella pertussis T cell immunity in humans
}

\author{
Maria Carollo a , Raffaella Palazzo ${ }^{a}$, Manuela Bianco $^{a}, K^{2}$ aatje Smits ${ }^{b}$, Françoise Mascart $^{b}$, \\ Clara Maria Ausielloa,* \\ a Department of Infectious, Parasitic and Immune-mediated Diseases, Istituto Superiore di Sanità, 00161 Rome, Italy \\ ${ }^{\mathrm{b}}$ Laboratory of Vaccinology and Mucosal Immunity, Université Libre de Bruxelles (U.L.B.), Brussels, Belgium
}

\section{A R T I C L E I N F O}

\section{Article history:}

Received 13 June 2011

Received in revised form

21 December 2011

Accepted 22 December 2011

Available online 9 January 2012

\section{Keywords:}

$\mathrm{T}$ cell responses

Th1 cytokines (IFN $\gamma$, TNF $\alpha$ )

Pertussis

Cryopreservation

5,6-Carboxylfluorescein diacetate

succinimidyl ester (CFSE) analysis

${ }^{3} \mathrm{H}$-thymidine incorporation

Blasts analysis

\begin{abstract}
A B S T R A C T
Measurement of antigen-specific $T$ cell responses is an adjunctive parameter to evaluate protection induced by a previous Bordetella pertussis infection or vaccination. The assessment of $\mathrm{T}$ cell responses is technically complex and usually performed on fresh peripheral blood mononuclear cells (PBMC). The objective of this study was to identify simplified methods to assess pertussis specific T cell responses and verify if these assays could be performed using frozen/thawed (frozen) PBMC.

Three read-outs to measure proliferation were compared: the fluorescent dye 5,6-carboxylfluorescein diacetate succinimidyl ester (CFSE) dilution test, the number of blast cells defined by physical parameters, and the incorporation of ${ }^{3} \mathrm{H}$-thymidine. The results of pertussis-specific assays performed on fresh PBMC were compared to the results on frozen PBMC from the same donor.

High concordance was obtained when the results of CFSE and blast read-outs were compared, an encouraging result since blast analysis allows the identification of proliferating cells and does not require any use of radioactive tracer as well as any staining.

The results obtained using fresh and frozen PBMC from the same donor in the different T cell assays, including IFN $\gamma$ and TNF $\alpha$ cytokine production, did not show significant differences, suggesting that a careful cryopreservation process of PBMC would not significantly influence $T$ cell response evaluation.

Adopting blast analysis and frozen PBMC, the possibility to test $\mathrm{T}$ cell responses is simplified and might be applied in population studies, providing for new instruments to better define correlates of protection still elusive in pertussis.
\end{abstract}

(C) 2011 Elsevier Ltd. All rights reserved.

\section{Introduction}

Measurement of antigen-specific T-cell response is a critical parameter used in various fields of lymphocyte research including evaluation of correlates of protection in vaccination and infection as well as in diagnosis of immunodeficiency disorder. Correlates of protection are still elusive in pertussis and evidences are collected that, beside antibody response, also $\mathrm{T}$ cell response plays a critical role in protection $[1,2]$. We decided to study $\mathrm{T}$ cell response to

Abbreviations: CD, cluster differentiation; CFSE, 5,6-carboxylfluorescein diacetate succinimidyl ester; cpm, counts per minute; FBS, Foetal Bovine Serum; FSC, forward scatter; FHA, filamentous hemagglutinin; frozen, frozen/thawed; PBMC, peripheral blood mononuclear cells; PBS, phosphate buffered saline; PI, proliferation index; PIw, proliferation index weighted; PT, pertussis toxin; SSC, side scatter; SEM, Standard Error of the Mean.

* Corresponding author at: Department of Infectious, Parasitic and Immunemediated Diseases, Anti-infectious Immunity Unit, Istituto Superiore di Sanità, Viale Regina Elena, 299, 00161 Rome, Italy. Tel.: +3906 4990 2890; fax: +39 0649903168.

E-mail address: clara.ausiello@iss.it (C.M. Ausiello). pertussis antigens since they are used in a phase I trial in humans to evaluate immunogenicity induced by intranasal administration of a new pertussis vaccine consisting in an attenuated Bordetella pertussis strain BPZE1 [3], within the ChildINNOVAC EU project (http://www.child-innovac.org).

Over the last thirty years, the "gold standard" method used to measure antigen-specific proliferation was the incorporation of ${ }^{3} \mathrm{H}$-thymidine in in vitro cell cultures. The level of ${ }^{3} \mathrm{H}$-thymidine incorporated correlates directly with the number of cells in $\mathrm{S}$ phase that synthesize the DNA during the pulse period of the incorporation [4-6]. This assay shows several disadvantages: primarily, it uses radioactive tracer, allowed only in restricted areas and implying major risks for the operators and the environment; secondly, it measures lymphocyte bulk proliferation at a specific time point, without providing for any information on the phenotype of the proliferating cells in a heterogeneous cell population, such as peripheral blood mononuclear cells (PBMC) and on the kinetics of proliferation, by assessing it only in the last $18 \mathrm{~h}$ of cultures.

Another well consolidated method to measure T-cell proliferation is based on the use of the fluorescent dye 
5,6-carboxyfluorescein diacetate succinimidyl ester (CFDA-SE, commonly named as CFSE). This is an alternative assay that allows the detection, identification and quantification of proliferating $\mathrm{T}$ cells [7]. CFSE is transported intra-cellularly during incubation of PBMC and then binds covalently to cytoplasmic proteins. During each cell division, intracellular CFSE concentration is halved in the daughter cells, allowing to monitor the mitotic activity of proliferating cells, easily identified by flow cytometry $[8,9]$. CFSE assay has been successfully applied to assess proliferation kinetics of both in vivo and in vitro activated cells in a variety of experimental settings [10,11]. Furthermore, proliferating cells can be characterized by using fluorescent-labeled antibodies to cluster differentiation (CD) antigens, which define specific subsets of lymphocytes. However, since CFSE is potentially toxic [12-14], the cells need extensive washes. This procedure might cause a loss in cell number, which could be a limiting factor for the assay when the amount of biological sample is poor, such as in neonates.

Given all above limits, we focused our attention on the blast analysis, a method performed initially using whole blood $[15,16]$ and more recently using isolated PBMC [6]. The method is based on flow cytometry analysis and on the electronic gating strategy, defining blasts as cells having greater forward scatter (FSC) and side scatter (SSC) than lymphocytes. This assay not only allows the identification and characterization of the proliferating cells by fluorescent-labeled antibodies to CD antigens, as in CFSE method, but also, not requiring the CFSE staining in the multi-parametric determination, leaves free the green fluorescence channel for another marker.

The first objective was to develop a T cells assay easy to perform, requiring low blood volume, measuring functional activity, highly sensitive in detecting low antigen-specific responses, without using radioactive tracer. To this aim, we investigated if pertussis-specific proliferation measured by blast analysis, which satisfy most of these criteria, correlates with CFSE analysis and also if these two flow cytometry read-outs correlate to ${ }^{3} \mathrm{H}$-thymidine assay.

The in vitro assays used to measure cellular immune functions are time consuming, technically complex, prone to variability, and usually performed in fresh specimens whose employment sensibly reduced the possibility to measure $T$ cell response in clinical trials or in population studies. The use of cryopreserved specimens would help to overcome these difficulties. Thus, the second objective was to evaluate if either fresh or frozen/thawed (frozen) human PBMC could be used for pertussis-specific $T$ cell response assessment.

Overall, the data obtained indicated the equivalence of results between the CFSE and blast analysis. A very good correlation was found between the two flow cytometry read-outs. The correlation with the ${ }^{3} \mathrm{H}$-thymidine incorporation was found only when pertussis toxin (PT) was used as antigen. Concerning the possibility of using cryopreserved PBMC, the proliferative capacity and the ability to secrete cytokine after antigenic stimulation of frozen PBMC was largely preserved. These results provide evidence that cryopreservation of PBMC may be appropriate for some retrospective analyses of immune function and for the use in clinical trials and population studies.

\section{Material and methods}

\subsection{Healthy donors}

A total of 29 healthy adults blood donors were tested for proliferation studies. A further group of 14 healthy adults blood donors was tested for comparing fresh and frozen PBMC. Heparinized peripheral blood was obtained from the Transfusional Centre of the University La Sapienza of Rome, Italy. This study was conducted according to the principles expressed in the Declaration of Helsinki. All blood donors provided written informed consent for the collection of samples and the subsequent analysis was processed anonymously.

\subsection{Reagents, antigens and mitogen}

Fluorescent dye CFSE was from Molecular Probes Europe BV (Leiden, the Netherlands). Pertussis vaccinal antigens PT and filamentous hemagglutinin (FHA) were purified starting from crude material obtained from Novartis, Siena, Italy, as described by Nasso and colleagues [17].

Before their use both proteins were heated inactivated $\left(96^{\circ} \mathrm{C} / 1 \mathrm{~h}\right.$ ) to avoid any mitogenicity induced by PT, even the slightest contamination in the FHA preparation $[18,19]$.

To stimulate PBMC, PT and FHA were used at the concentration of $5 \mu \mathrm{g} / \mathrm{ml}$ and $10 \mu \mathrm{g} / \mathrm{ml}$, respectively, on the basis of a doseresponse curve published in a previous study [20]. As a positive control of the $\mathrm{T}$ cell responses, stimulation induced by the superantigen Staphylococcus Enterotoxin B $(1 \mu \mathrm{g} / \mathrm{ml})$ (Sigma Chemical Co., St. Louis, MO, USA) was used.

\subsection{PBMC isolation and cell culture}

PBMC were isolated on a density gradient with Lympholyte $\mathrm{H}$ Cell Separation Medium (Cedarlane Lab Limited, Hornby, Ontario, Canada), washed twice in RPMI 1640 medium (Gibco, Gran Island, NY, USA). Cells were seeded at the density of $1 \times 10^{6}$ cells $/ \mathrm{ml}$ in RPMI 1640 (Gibco), supplemented with antibiotics $(100 \mathrm{U} / \mathrm{ml}$ of penicillin, $0.1 \mu \mathrm{g} / \mathrm{ml}$ of streptomycin) $0.1 \mathrm{mM}$ non-essential amino acids, $1 \mathrm{mM}$ sodium pyruvate, $2 \mathrm{mM}$ L-glutamine, $25 \mathrm{mM}$ HEPES, all from Euroclone, and $0.05 \mathrm{mM}$ 2-mercaptoethanol (Sigma). Medium was supplemented with 10\% LPS-screened Foetal Bovine Serum (FBS) (Hyclone Laboratories, South Logan, UT) or 5\% pooled human serum (Blutspendezentrum Universitätsspital, Basel, Switzerland) [20].

PBMC were used either as freshly isolated or frozen after cryopreservation. In the latter case, fresh isolated PBMC were suspended in cold FBS and left on ice for $15 \mathrm{~min}$, then an equal amount of cold FBS supplemented with $20 \%$ of dimethyl sulfoxide (Sigma) was added to the cells, immediately frozen at the density of 10-20 millions $/ 500 \mu \mathrm{l} / \mathrm{vial}$ in Mr. Frosty box (Nalgene) and stored at $-80^{\circ} \mathrm{C}$, then the vials were transferred in liquid nitrogen. On the day of the experiment, the vial was quickly thawed at $37^{\circ} \mathrm{C}$, washed in RPMI 1640 and then processed exactly as fresh PBMC.

\subsection{PBMC proliferation assays}

Three different read-outs were used to evaluate PBMC proliferation.

\subsection{1. ${ }^{3}$ H-thymidine incorporation}

Cells were seeded at the concentration of $2 \times 10^{5}$ cells $10.2 \mathrm{ml} /$ well in complete medium supplemented with $5 \%$ pooled human serum, in triplicates, in flat-bottom 96-well microplates (Falcon, Becton Dickinson Labware, Europe) with or without stimuli. Plates were incubated at $37{ }^{\circ} \mathrm{C}$ in a humidified $5 \% \mathrm{CO}_{2}$ atmosphere for 6 days. DNA synthesis was evaluated by incorporation of ${ }^{3} \mathrm{H}$-thymidine $(0.5 \mu \mathrm{Ci} /$ well, Amersham Biosciences, United Kingdom), measured after the last $18 \mathrm{~h}$ of culture. Data were expressed as counts per minute $(\mathrm{cpm}) \times 10^{3}[20]$.

\subsubsection{Analysis of CFSE and the blast population: flow cytometric} data acquisition and analysis

PBMC were suspended in warm sterile phosphate buffered saline (PBS) and CFSE was added at the final concentration of $5 \mu \mathrm{M}$. Cell suspension was vortexed immediately following CFSE addition, 

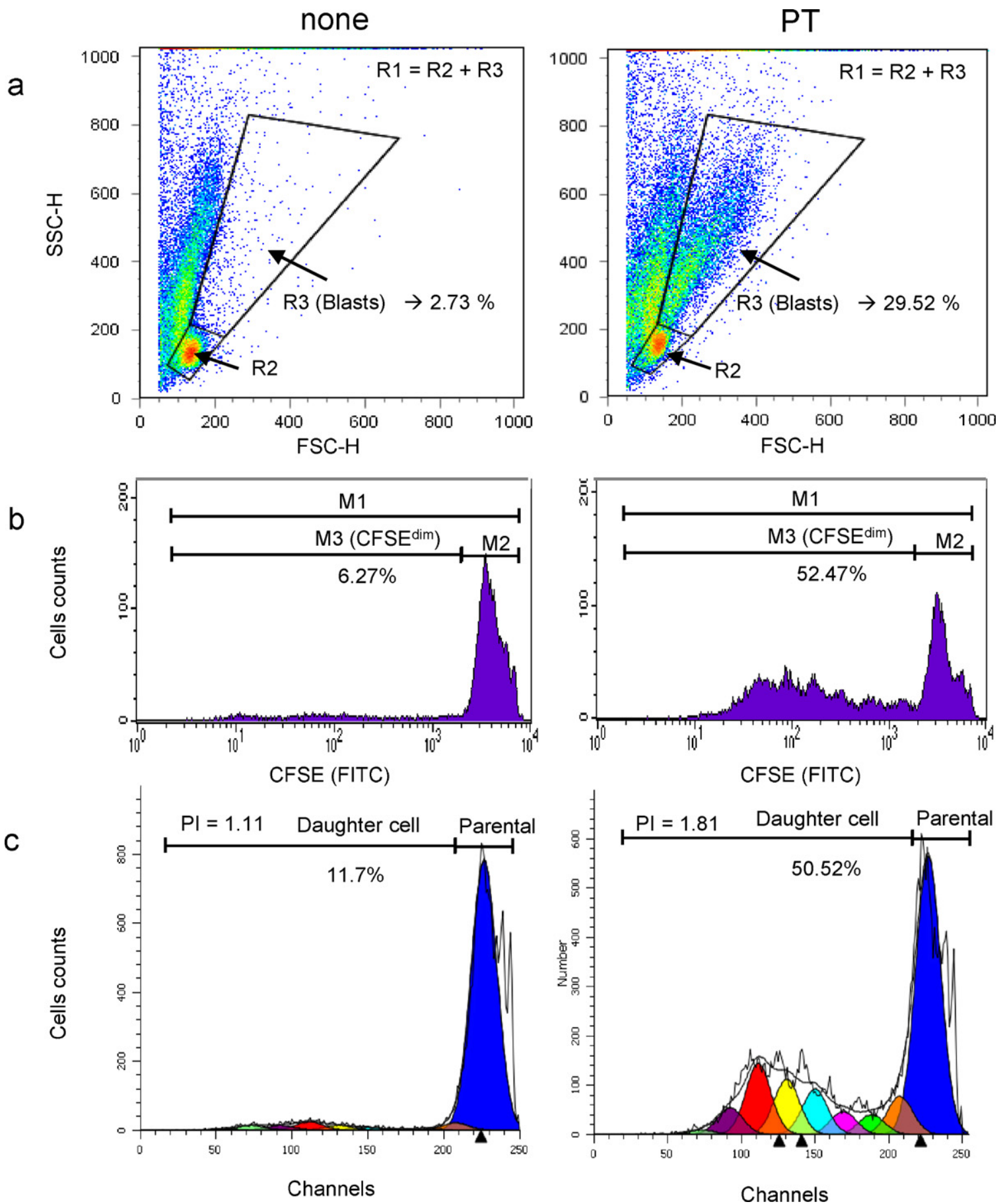

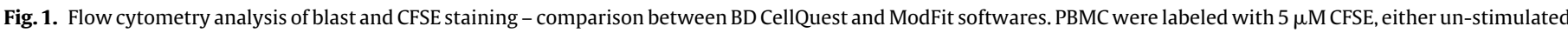

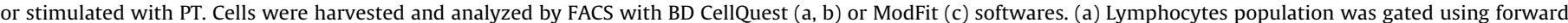

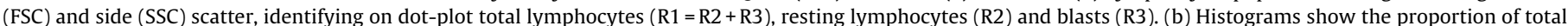

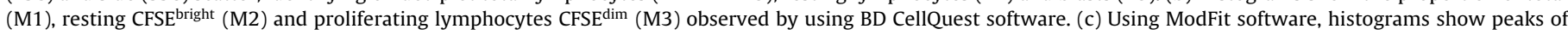
parental undivided PBMC, percentage of daughter cells and the proliferation index (PI).

and then incubated for $4 \mathrm{~min}$ at room temperature in a dark place. The labeled cells were washed twice with RPMI 1640 medium supplemented with $10 \%$ FBS in cold conditions. Cells were suspended at the density of $1 \times 10^{6} / \mathrm{ml}$ in complete medium supplemented with $10 \%$ of FBS and co-stimuli CD28 and CD49d $(1 \mu \mathrm{g} / \mathrm{ml})(\mathrm{BD}$ Biosciences, San Jose, CA, USA). After 4 days of culture, cells were washed in PBS, fixed in paraformaldehyde (4\%) for $10 \mathrm{~min}$ at room temperature and analyzed on a FACS Scan (BD Biosciences).
Fig. 1 shows the gate strategy in a representative experiment where CFSE and blast analysis in un-stimulated- or PT-activated PBMC cultures were compared. Total lymphocytes were electronically gated in R1 using FSC and SSC properties; cellular debris and granular cells were excluded (Fig. 1, panel a). A total of $20 \times 10^{3}$ events in the total lymphocyte gate (R1) were acquired for each sample. By means of FSC and SSC properties, the total lymphocytes (R1), the undivided (R2) and the proliferating cells (blasts, R3) 
were defined [15]. As shown in Fig. 1, panel b, the cells in M2 with a higher fluorescence (CFSE ${ }^{\text {bright }}$ ) and the cells in M3 with lower fluorescence (CFSE ${ }^{\mathrm{dim}}$ ) were defined as undivided or proliferating lymphocytes. CellQuest software was used to perform the analysis (BD Biosciences) (Fig. 1, panel b).

As shown in Fig. 1, panel c, the frequency of proliferating cells was also evaluated by using the Proliferation Wizard module of ModFit LT software (Verity Software House, Version 2, Topsham, ME), based on mathematical deconvolution model. Using this model, the number of parental and daughter cells, the proliferation index (PI), as well as the proliferation index weighted (PIw), calculated multiplying the value of PI by daughter cells, were determined (Fig. 1, panel c). The ModFit software takes in consideration the percentage of proliferating cells going from the third round of cell division, since it has been hypothesized that true proliferation starts from the third generation $[21,22]$.

Using these analyses, proliferating cells were identified as percentage of blasts by physical parameter in R3 (Fig. 1, panel a) or percentage of CFSE ${ }^{\text {dim }}$ cells in M3 (Fig. 1, panel b) or PI and PIw (Fig. 1, panel c).

\subsection{Pertussis antigen-specific immune response by intracellular cytokine production}

PBMC were seeded at the concentration of $5 \times 10^{5}$ in $500 \mu \mathrm{l}$ into $5 \mathrm{ml}$ Falcon ${ }^{\mathrm{TM}}$ polystyrene tube and added with co-stimuli CD28 and CD49d $(1 \mu \mathrm{g} / \mathrm{ml})$ (BD Biosciences) and with PT, FHA or appropriate negative (medium) or positive (Staphylococcus Enterotoxin B) controls. Tubes were incubated at $37^{\circ} \mathrm{C}$ in $5 \% \mathrm{CO}_{2}$ for 5 days. Brefeldin A (Sigma) was added during the last $4 \mathrm{~h}$ of culture. Cells were washed in PBS, fixed in $1 \mathrm{ml}$ paraformaldehyde $2 \%$ for $15 \mathrm{~min}$ and washed in staining buffer (PBS/Bovine Serum Albumine 0.5\%/Sodium Azide 0.02\%) [23]. Cells were simultaneously stained for extracellular markers (CD4, CD8) and intracellular cytokines (IFN $\gamma$ and TNF $\alpha$ ), using mouse anti-human CD4-PercCPCy5.5, CD8-APC-Cy ${ }^{\mathrm{TM}} 7$, IFN $\gamma$ Alexa Fluor 647 and TNF $\alpha$-FITC, all purchased from BD Biosciences and diluted in staining buffer plus $0.5 \%$ saponin. All samples were incubated for $30 \mathrm{~min}$ in the dark at room temperature, washed in staining buffer and processed for flow cytometric analysis on a FACSCanto (BD Biosciences).

\subsection{Statistical analysis}

All data were recorded in a computerized database. Results are reported as means \pm Standard Error of the Mean (SEM). Statistical analysis was carried out using SPSS (version 17.0; SPSS Inc, Chicago, IL) software. Differences within groups obtained by the different assays were evaluated by the Wilcoxon paired-samples test. Values of $p$ less than 0.05 were considered to indicate a statistical significance, and all reported $p$ values were two-sided.

When appropriate, a linear regression model was applied and the Pearson's correlation coefficient $(R)$ was calculated.

\section{Results}

\subsection{Pertussis antigen-specific proliferation in PBMC}

The pertussis specific proliferation evaluated by blasts based on size analysis of the cells, by CFSE dilution and by ${ }^{3} \mathrm{H}$-thymidine incorporation using PBMC from 29 subjects were compared. The descriptive statistical analysis and the linear regression analysis of the data obtained and the correlation coefficient ( $R$ values) were calculated and shown in Table 1.

A highly significant correlation between CFSE $^{\text {dim }}$ and blast percentage in PT- or FHA-stimulated and un-stimulated PBMC was obtained by using the CellQuest software.
Proliferation data were also analyzed by using the ModFit software as described in Fig. 1.

As shown in Table 1, the blast percentage was compared to PI or PIw and a significant correlation was found when PBMC were stimulated with PT or FHA, according to results previously shown, but the $R$ values were in general lower than those obtained with CellQuest analysis (Table 1).

On the basis of the best correlation given by the CellQuest software, we selected this software to analyze all data.

Thereby, we compared results of specific $\mathrm{T}$ cell proliferation obtained by blasts or CFSE ${ }^{\text {dim }}$ with those obtained with ${ }^{3} \mathrm{H}$ thymidine incorporation (Table 1). A significant correlation was found in PT-stimulated PBMC when the three read-outs were compared, even though the $R$ value between proliferations measured with ${ }^{3} \mathrm{H}$-thymidine/CFSE ${ }^{\mathrm{dim}}(R=0.691)$ and ${ }^{3} \mathrm{H}$-thymidine/Blast $(R=0.688)$ was lower with respect to the correlation between Blast/CFSE $^{\mathrm{dim}}(R=0.919)$ (Table 1$)$. No significant correlation was found in FHA-stimulated or un-stimulated PBMC when blasts or CFSE $^{\text {dim }}$ results were compared to those ones with ${ }^{3} \mathrm{H}$-thymidine incorporation.

We concluded that proliferation measured by blast analysis could be a good alternative to the CFSE read-out and, depending on the antigen, also to the ${ }^{3} \mathrm{H}$-thymidine incorporation.

\subsection{Pertussis specific T-cell proliferation in fresh versus frozen PBMC}

The use of cryopreserved PBMC would increase the efficiency of $T$ cell assays in clinical trials and in population studies where specimens are collected over many months and at multiple locations. More importantly, the possibility to test multiple specimens obtained over a period of time from the same donor in the same assay would reduce within-donors variability [24], facilitating the measurements of $\mathrm{T}$ cell functions. To this aim, we performed a comparative analysis of pertussisspecific $\mathrm{T}$ cell proliferation in frozen PBMC compared to fresh PBMC.

We first compared the results of both blast and CFSE readouts on frozen PBMC obtained from 14 blood donors. As shown in Fig. 2, panel a, the regression analysis generates a significant $R$ coefficient when proliferative responses were measured as percentage of CFSE ${ }^{\text {dim }}$ versus blasts in PT- and FHA-stimulated and un-stimulated PBMC, indicating a high concordance between the two read-outs. These results parallel the data obtained with fresh PBMC shown in Table 1, even though the $R$ values in PT-stimulated cultures were lower $(R=0.919$ in fresh versus $R=0.751$ in frozen cultures).

We further compared the antigen-specific T-cell proliferation in fresh versus frozen PBMC obtained from the same donor. Ten pair data were matched and, as shown in Fig. 2 (panels b for blast read-out and $\mathrm{c}$ for CFSE read-out), not significant differences were found with data analyzed by the Wilcoxon paired-samples test. Moreover, a good correlation was found for PT and FHA stimuli when blast read-out was used (Fig. 2b), indicating that frozen PBMC can be used instead of fresh cells. When the proliferative response was evaluated by CFSE $^{\text {dim }}$, a good $R$ was found only in PT-activated PBMC (Fig. 2c).

These data confirmed that it is possible to use frozen PBMC when the proliferation is assessed by flow cytometry and that once again the blast analysis showed a better correlation with respect to CFSE read-out. 
a

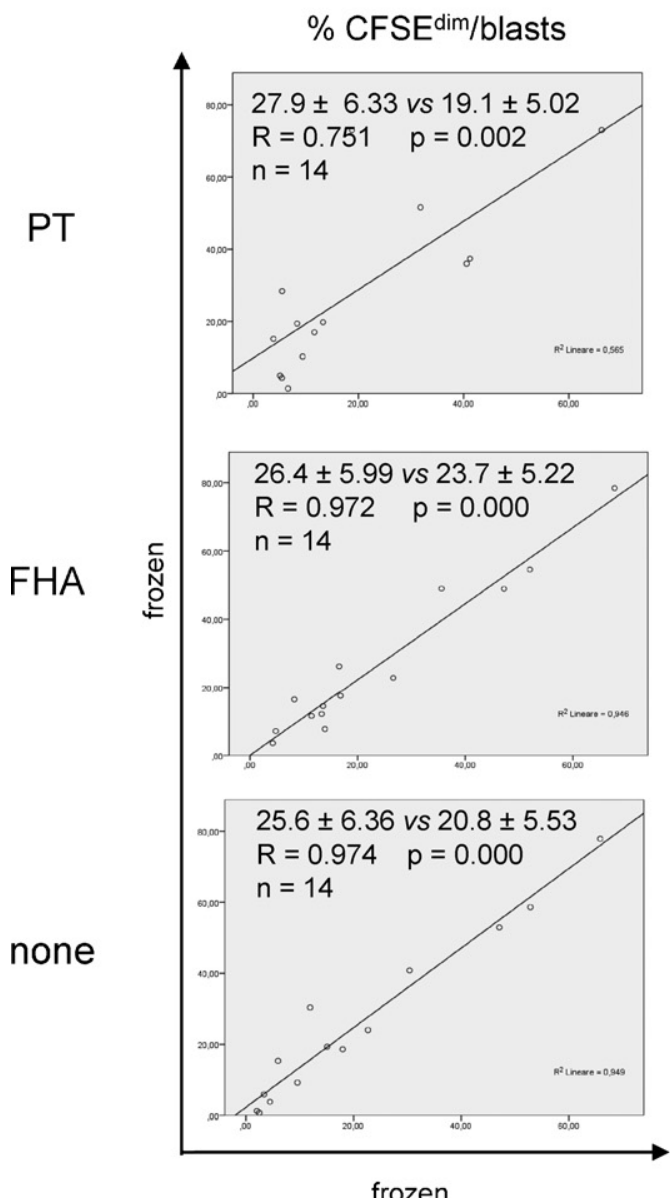

b

$\%$ blasts
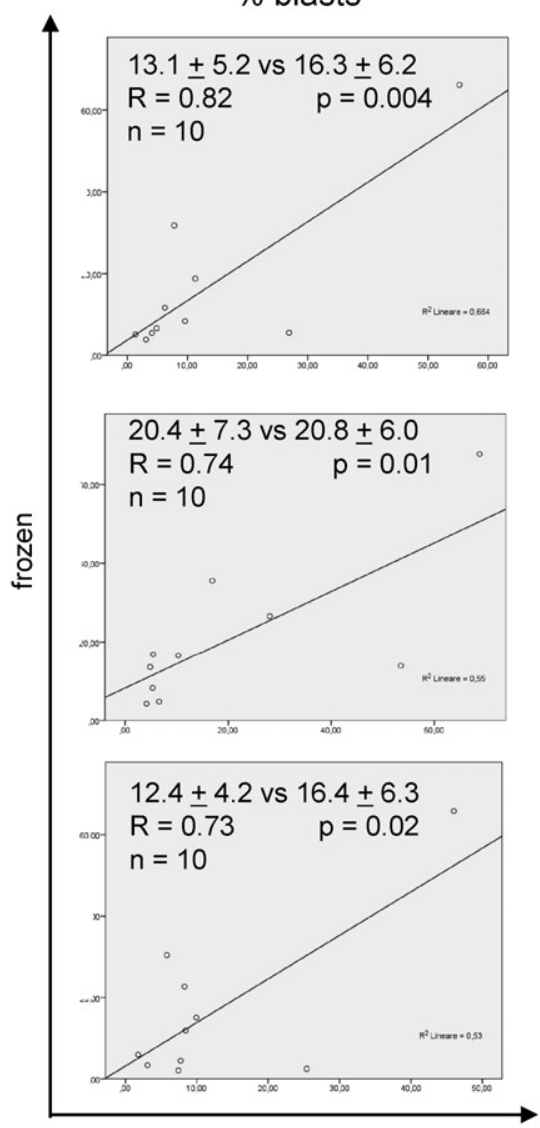

fresh
C

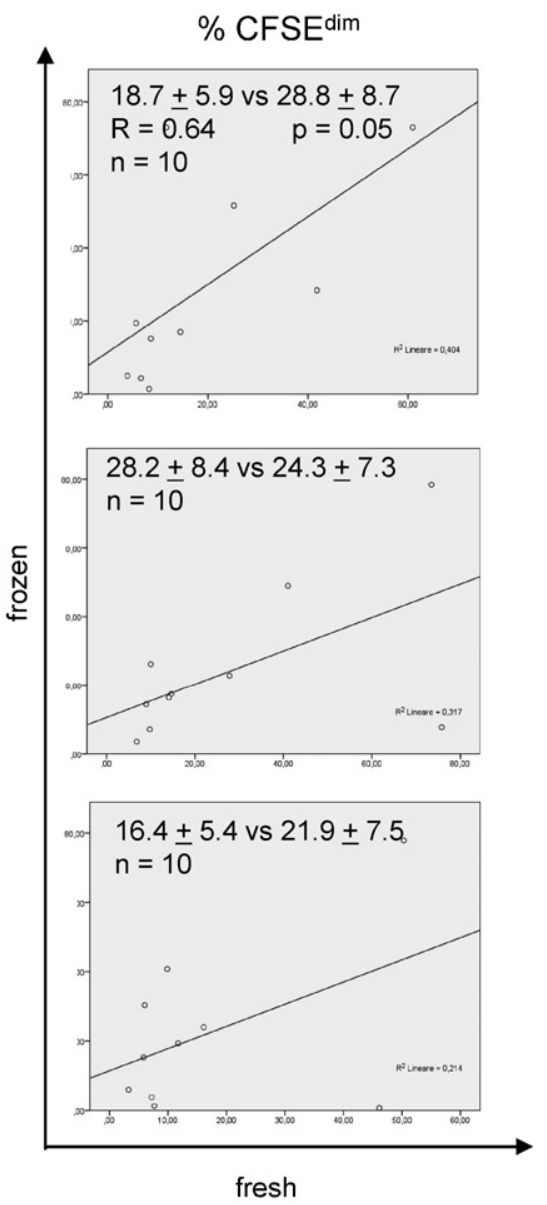

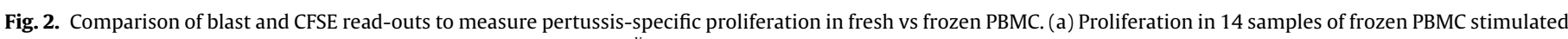

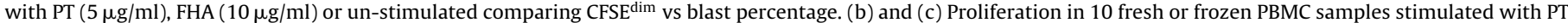

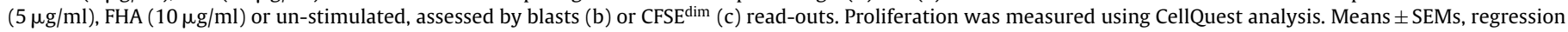
analysis, Pearson's correlation $(R)$ coefficient and $p$ values are shown.

IF $N \gamma$

\section{PT}
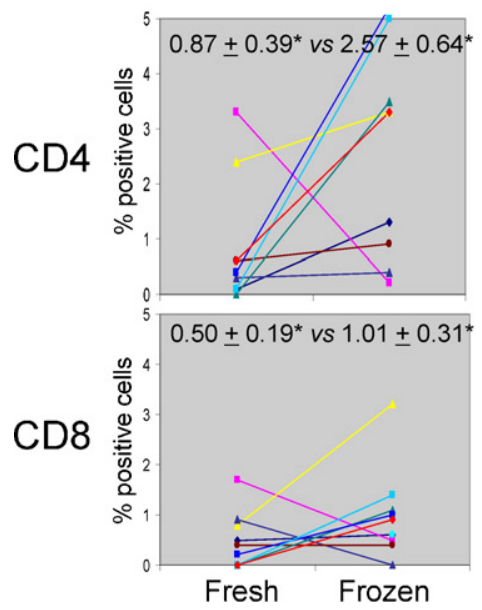

FHA
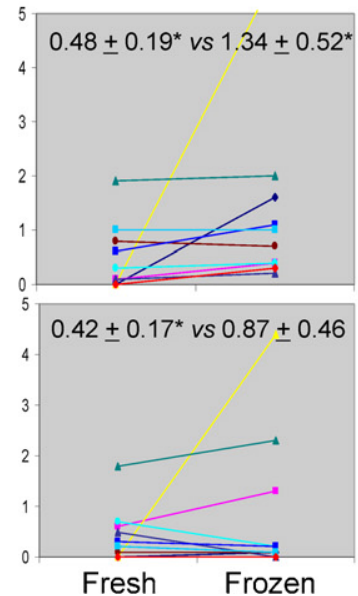

$\mathrm{TNF} \alpha$

PT
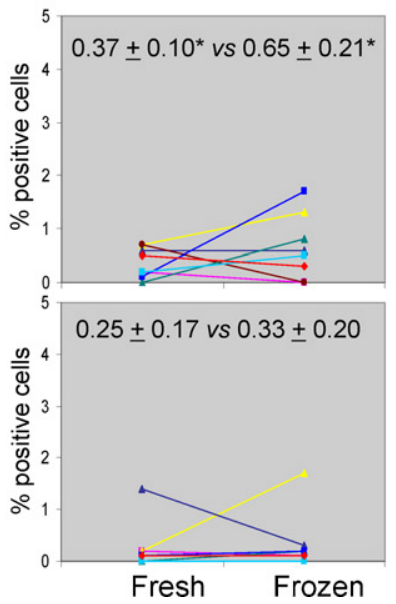

FHA

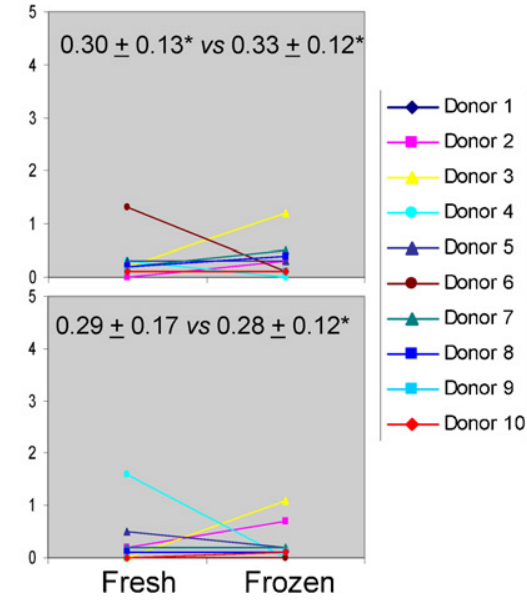

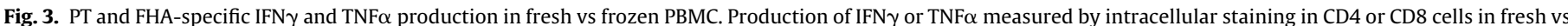

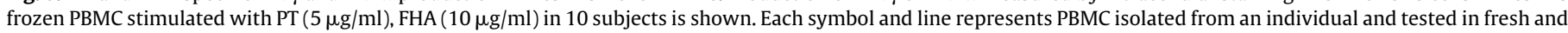

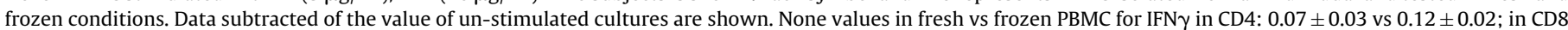

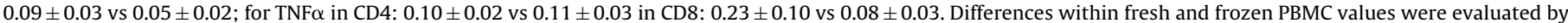

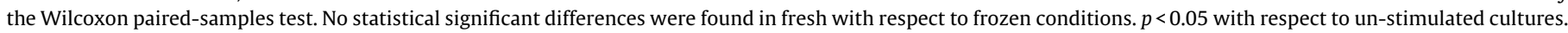


Table 1

Comparison of blast, CFSE and ${ }^{3} \mathrm{H}$-thymidine read-outs to measure pertussis-specific proliferation in PBMC.

\begin{tabular}{|c|c|c|c|}
\hline Parameters & PT & FHA & None \\
\hline$\%$ CFSE $^{\text {dim }}$ vs \% blasts & $\begin{array}{l}20.1 \pm 3.8 \text { vs } 13.5 \pm 3.3 \\
R=0.919, p=0.000 \\
n=27\end{array}$ & $\begin{array}{l}27.9 \pm 5.3 \text { vs } 19.9 \pm 4.6 \\
R=0.955, p=0.000 \\
n=25\end{array}$ & $\begin{array}{l}15.8 \pm 3.4 \text { vs } 11.5 \pm 2.8 \\
R=0.913, p=0.000 \\
n=29\end{array}$ \\
\hline PI vs \% blasts & $\begin{array}{l}1.1 \pm 0.06 \text { vs } 6.8 \pm 2.2 \\
R=0.906, p=0.000 \\
n=18\end{array}$ & $\begin{array}{l}1.1 \pm 0.04 \text { vs } 7.1 \pm 1.7 \\
R=0.646, p=0.007 \\
n=16\end{array}$ & $\begin{array}{l}1.1 \pm 0.01 \text { vs } 5.1 \pm 0.8 \\
R=0.213, p=0.366 \\
n=20\end{array}$ \\
\hline PIw vs \% blasts & $\begin{array}{l}18.4 \pm 6.8 \text { vs } 6.8 \pm 2.2 \\
R=0.893, p=0.000 \\
n=18\end{array}$ & $\begin{array}{l}16.2 \pm 3.9 \text { vs } 7.1 \pm 1.7 \\
R=0.640, p=0.008 \\
n=16\end{array}$ & $\begin{array}{l}8.2 \pm 1.5 \text { vs } 5.1 \pm 0.8 \\
R=0.130, p=0.584 \\
n=20\end{array}$ \\
\hline$\% \mathrm{CFSE}^{\mathrm{dim}} \mathrm{vs}^{3} \mathrm{H} \mathrm{cpm}$ & $\begin{array}{l}23.2 \pm 4.7 \text { vs } 8.0 \pm 2.5 \\
R=0.691, p=0.001 \\
n=20\end{array}$ & $\begin{array}{l}29.2 \pm 6.1 \text { vs } 3.9 \pm 1.7 \\
R=0.198, p=0.430 \\
n=18\end{array}$ & $\begin{array}{l}15.3 \pm 3.6 \text { vs } 0.7 \pm 0.1 \\
R=0.041, p=0.849 \\
n=24\end{array}$ \\
\hline$\%$ blasts vs ${ }^{3} \mathrm{H} \mathrm{cpm}$ & $\begin{array}{l}15.3 \pm 4.1 \text { vs } 8.0 \pm 2.5 \\
R=0.688, p=0.001 \\
n=20\end{array}$ & $\begin{array}{l}20.6 \pm 5.6 \text { vs } 3.9 \pm 1.7 \\
R=0.238, p=0.342 \\
n=18\end{array}$ & $\begin{array}{l}11.4 \pm 3.3 \text { vs } 0.7 \pm 0.1 \\
R=0.026, p=0.903 \\
n=24\end{array}$ \\
\hline
\end{tabular}

a PBMC from indicated numbers ( $n$ ) of adults blood donors were stimulated with PT ( $5 \mu \mathrm{g} / \mathrm{ml})$, FHA ( $10 \mu \mathrm{g} / \mathrm{ml})$ or left un-stimulated for 4 days in presence of costimul

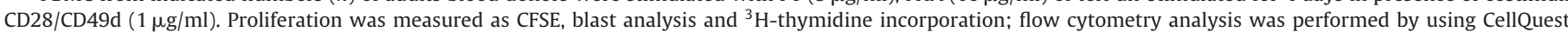

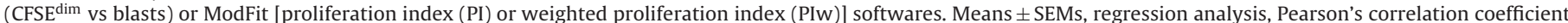
$(R)$ and $p$ values are shown.

\subsection{Pertussis-specific IFN $\gamma$ and TNF $\alpha$ production in fresh versus frozen PBMC}

Lymphocyte proliferation represents only one parameter of antigen-induced $\mathrm{T}$ cell activation, since the analysis of cytokine induction is important for a complete characterization of $\mathrm{T}$ cell functions. IFN $\gamma$ is known to be induced either after $B$. pertussis infection or vaccination and the induction of Th1 response is considered protective in pertussis [1,2,25-29]. To discover whether this parameter could be similarly evaluated in fresh and in frozen PBMC, we investigated both CD4 and CD8 T lymphocytes, able to produce IFN $\gamma$ [42]. In addition, we studied the production of TNF $\alpha$, a cytokine secreted after Th1 activation [30].

Using fresh or frozen cells, we found the significant increase of IFN $\gamma$ in both CD4 and CD8 cells stimulated with $B$. pertussis antigens compared to un-stimulated cultures, whereas a significant increase in TNF $\alpha$ production with respect to un-stimulated cells was observed only in CD4 cells (Fig. 3 ).

Freezing procedures did not affect significantly the IFN $\gamma$ and TNF $\alpha$ production, either in PT- or FHA-stimulated PBMC, when the fresh/frozen paired data were analyzed by the Wilcoxon pairedsamples test (Fig. 3). However, the percentages of cytokine positive cells measured in fresh versus frozen cells from the same subject showed a great variability. In general, a trend for a higher cytokine production was found in frozen versus fresh PBMC cultures, mainly in the IFN $\gamma$ production.

\section{Discussion}

Our findings showed that flow cytometry assay can be successfully used to evaluate pertussis antigen induced proliferation in PBMC from blood donors. A high concordance between the results of CFSE and blast read-outs was obtained. The comparison of proliferation data obtained between the two flow cytometry analyses and the ${ }^{3} \mathrm{H}$-thymidine incorporation needs to be more deeply investigated, based on a significant correlation between ${ }^{3} \mathrm{H}$-thymidine and blast- or CFSE-data, only when PT was used as antigen. Several authors found good concordance when PBMC proliferation was measured by CFSE assay or ${ }^{3} \mathrm{H}$-thymidine incorporation in both mitogenic and antigenic stimulations [14,24,31-33]. Among all studies on the evaluation of antigen specific responses, Angulo and Fulcher found good correlation in Candida-specific blastogenesis, particularly when the proliferation was induced by high Candida antigen concentration, and in PBMC from subjects with episodes of vaginal candidiasis $[32,34]$. Furthermore, Moore and colleagues found good agreement between the proliferation index of the CFSE assay and the stimulation index of the ${ }^{3} \mathrm{H}$-thymidine assay when lymphocyte responses to rabies virus were measured in vaccinated subjects [21]. Similarly, when Cytomegalovirus antigen specific proliferation was performed in PBMC cultures, there was a good concordance between the proliferative responses measured by ${ }^{3} \mathrm{H}-$ thymidine assay and blast assays performed in non HIV-infected controls, while in the HIV-infected low responders the concordance decreased [35].

The analysis of these published studies suggested that a better correlation between ${ }^{3} \mathrm{H}$-thymidine uptake and CFSE assays was reached in strong antigenic responses. In this study, we did not select PBMC donors on the basis of their responsiveness to pertussis antigens because, in a previous study, we found more than $80 \%$ of adults were responsive to PT, this probably due to a high pertussis circulation in our country by the end of 2000 [20,36]. However, in this study we discovered that, while adopting the same criteria (net $\mathrm{cpm}>3.000$, calculated by subtracting cpm of untreated PBMC cultures from cpm of the stimulated ones), the responsiveness to PT stimulation in blood donors was rather low; indeed around 60\%. This result probably is due to a low incidence of pertussis in Italy, a sharp decline expected from a higher vaccine coverage $(88 \%$ in 1998 to $95 \%$ in 2003). Likewise, pertussis incidence rate declined from 19.0 (1990-1996) to 6.6 (1997-2002) per 100,000 inhabitants [37], reaching the incidence of 0.6 per 100,000 inhabitants in 2008 (www.euvac.net). The response to FHA was even lower. Therefore, a relative stronger proliferative response to PT antigen may explain the good correlation between the two flow cytometry read-outs and ${ }^{3} \mathrm{H}$-thymidine incorporation only in PT specific response.

Flow cytometry assays are more suitable to measure low antigenic responses as demonstrated by Mannering et al.; indeed CFSE assay was more sensitive than ${ }^{3} \mathrm{H}$-thymidine incorporation to detect the proliferation of rare antigen-specific CD4 $\mathrm{T}$ cells at 10-fold lower antigen concentrations [5]. Besides, Venken and colleagues, measuring Treg suppressive capacity, demonstrated that CFSE assay allowed the detection of a significant Treg suppression whereas this was clearly underestimated using the ${ }^{3} \mathrm{H}$-thymidine assay [38].

Overall, our data demonstrated that it is possible to evaluate pertussis-specific proliferative $\mathrm{T}$ cells response by simply using the morphological parameters to identify the blast region. This 
read-out can be used as an alternative to CFSE staining with benefits of saving time in skipping CFSE cell labeling step, and in the preparation of samples, since there is not need to wash the cells extensively to eliminate the toxic effect of CFSE dye [14].

In addition, the flow cytometry analysis appears to be more sensitive and can be used also to detect low proliferative responses. It is important to underline that the flow cytometry allows further characterization of the proliferating cells by phenotype analysis for a more comprehensive evaluation of the role T cells in immune response.

The results obtained using frozen PBMC to measure antigenic induced proliferation are consistent with the results obtained in fresh PBMC. Here again no significant differences were found in the mean values of proliferation induced by pertussis antigens in fresh or frozen PBMC from the same donor and also a clear correlation was obtained when CFSE and blast read-outs were compared (Fig. $2 \mathrm{~b}$ and $\mathrm{c}$ ), but a very good correlation was found only in blast assays regardless the stimulus used, indicating that this read-out is more robust and independent from the cryopreservation procedures. When comparing the CFSE results in fresh and frozen PBMC from the same donor, a significant correlation was found only in PT stimulated cultures and was rather low. These data again confirm that blast read-out is better than CFSE read-out to measure antigen specific subsets after antigen stimulation.

IFN $\gamma$ production resulted significantly enhanced after PT and FHA stimulation in CD4 and CD8 and in fresh and frozen cells with respect to un-stimulated control cells. The only exception was the production of IFN $\gamma$ in frozen CD8 cells. This indicates that the antigen stimulation was effective.

However, although there were no significant differences between the results obtained in fresh and frozen cells from the same donor, within-donor variability was observed. In general, a tendency to an increase of antigen induced cytokine production in cultures derived from frozen PBMC was noticed, suggesting the existence of a regulatory cell population eliminated by the cryopreservation procedures. Evidences that cryopreservation affects $\mathrm{T}$ regulatory function are reported in several papers [39-41]. To validate this hypothesis further studies should be implemented aiming to demonstrate an effective reduction of $T$ regulatory cells in our freezing conditions and that this reduction is responsible for a higher cytokine induction.

Our data are only partially in keeping with the data reported by Owen and colleagues [35] demonstrating that long-term cryopreservation had detrimental effects on T cell IFN $\gamma$ responses in HIV infected individuals, but the loss of responses to Cytomegalovirus (CMV) peptide pools were minimal in HIV-negative individuals. Indeed, our cells were kept frozen for no longer than three months, beside the fact that PBMC were obtained from healthy subjects. Moreover, Owen and colleagues did not directly compare fresh and short-term cryopreserved PBMC [35].

In conclusion, this study indicates that blast analysis and frozen PBMC simplify the testing on $T$ cell responses which can be applied in population studies, thus giving new instruments to better define correlates of protection still elusive in pertussis.

\section{Acknowledgements}

We thank Giuseppina Mandarino for the help in revision and editing of the manuscript and Giorgio Fedele and Rigmor Thorstensson for critically reading of the manuscript.

Contributors: Conceived and designed the experiments: MC, CMA; Performed the experiments: MC, RP, MB; Analyzed the data: MC, RP, CMA; Revised the manuscript critically for important intellectual content: KS, FM; Wrote the paper: MC, CMA. Conflict of interest statement: No author has any financial, personal, or professional interests that could be construed to have influenced the paper. Funding: This work was supported by grants from AIFA (Agenzia Italiana del Farmaco, code FAM67NFPN), from the European Commission under the grant agreement \#201502 (ChildINNOVAC) and by the Italian Ministry of Health, CCM (1M51). The funders had no role in study design, data collection and analysis, decision to publish, or preparation of the manuscript.

\section{References}

[1] Mills KH. Immunity to Bordetella pertussis. Microbes Infect 2001;3:655-77.

[2] de Gouw D, Diavatopoulos DA, Bootsma HJ, Hermans PW, Mooi FR. Pertussis: a matter of immune modulation. FEMS Microbiol Rev 2011;35:441-74.

[3] Mielcarek N, Debrie AS, Raze D, Bertout J, Rouanet C, Younes AB, et al. Live attenuated $B$. pertussis as a single-dose nasal vaccine against whooping cough. PLoS Pathog 2006;2:e65.

[4] Piguet PF, Vassalli P. Study of the thymic-derived or -independent nature of mouse spleen cells induced to proliferate in culture by various mitogens and antigens. Eur J Immunol 1973;3:477-83.

[5] Mannering SI, Morris JS, Jensen KP, Purcell AW, Honeyman MC, van Endert PM, et al. A sensitive method for detecting proliferation of rare autoantigen-specific human T cells. J Immunol Methods 2003;283:173-83.

[6] Aboud S, Nilsson C, Karlén K, Marovich M, Wahren B, Sandström E, et al. Strong HIV-specific CD4+ and CD8+ T-lymphocyte proliferative responses in healthy individuals immunized with an HIV-1 DNA vaccine and boosted with recombinant modified vaccinia virus ankara expressing HIV-1 genes. Clin Vaccine Immunol 2010;17:1124-31.

[7] Lyons AB, Parish C. Determination of lymphocyte division by flow cytometry. Immunol Methods 1994;171:131-7.

[8] Bernard S, Pujo-Menjouet L, Mackey MC. Analysis of cell kinetics using a cell division marker: mathematical modeling of experimental data. Biophys J 2003;84:3414-24.

[9] Nordon RE, Nakamura M, Ramirez C, Odell R. Analysis of growth kinetics by division tracking. Immunol Cell Biol 1999;77:523-9.

[10] Chen JC, Chang ML, Muench MO. A kinetic study of the murine mixed lymphocyte reaction by 5,6-carboxyfluorescein diacetate succinimidyl ester labeling. J Immunol Methods 2003;279:123-33.

[11] Dengler TJ, Johnson DR, Pober JS. Human vascular endothelial cells stimulate a lower frequency of alloreactive CD8+ pre-CTL and induce less clonal expansion than matching B lymphoblastoid cells: development of a novel limiting dilution analysis method based on CFSE labeling of lymphocytes. J Immunol 2001:166:3846-54.

[12] Givan AL, Fisher JL, Waugh MG, Bercovici N, Wallace PK. Use of cell-tracking dyes to determine proliferation precursor frequencies of antigen-specific $\mathrm{T}$ cells. Methods Mol Biol 2004;263:109-24.

[13] Quah BJ, Warren HS, Parish CR. Monitoring lymphocyte proliferation in vitro and in vivo with the intracellular fluorescent dye carboxyfluorescein diacetate succinimidyl ester. Nat Protoc 2007;2:2049-56.

[14] Last'ovicka J, Budinský V, Spísek R, Bartůnková J. Assessment of lymphocyte proliferation: CFSE kills dividing cells and modulates expression of activation markers. Cell Immunol 2009;256:79-85.

[15] Gaines H, Andersson L, Biberfeld G. A new method for measuring lymphoproliferation at the single-cell level in whole blood cultures by flow cytometry. J Immunol Methods 1996;195:63-72.

[16] Svahn A, Linde A, Thorstensson R, Karlén K, Andersson L, Gaines H. Development and evaluation of a flow-cytometric assay of specific cell-mediated immune response in activated whole blood for the detection of cell-mediated immunity against varicella-zoster virus. J Immunol Methods 2003;277:17-25.

[17] Nasso M, Fedele G, Spensieri F, Palazzo R, Costantino P, Rappuoli R, et al. Genetically detoxified pertussis toxin induces Th1/Th17 immune response through MAPKs and IL-10-dependent mechanisms. J Immunol 2009;183:1892-9.

[18] Gray LS, Huber KS, Gray MC, Hewlett EL, Engelhard VH. Pertussis toxin effects on T lymphocytes are mediated through $\mathrm{CD} 3$ and not by pertussis toxin catalyzed modification of a G protein. J Immunol 1989;142:1631-8.

[19] Nencioni L, Pizza MG, Volpini G, De Magistris MT, Giovannoni F, Rappuoli R Properties of the B oligomer of pertussis toxin. Infect Immun 1991:59:4732-4

[20] Ausiello CM, Lande R, la Sala A, Urbani F, Cassone A. Cell-mediated immune response of healthy adults to Bordetella pertussis vaccine antigens. J Infect Dis 1998; 178:466-70.

[21] Moore SM, Wilkerson MJ, Davis RD, Wyatt CR, Briggs DJ. Detection of cellular immunity to rabies antigens in human vaccinees. J Clin Immunol 2006;26:533-45.

[22] Liu D, Yu J, Chen $\mathrm{H}$, Reichman $\mathrm{R}, \mathrm{Wu} \mathrm{H}$, Jin X. Statistical determination of threshold for cellular division in the CFSE-labeling assay. J Immunol Methods 2006;312:126-36.

[23] Palazzo R, Spensieri F, Massari M, Fedele G, Frasca L, Carrara S, et al. Use of whole-blood samples in in-house bulk and single-cell antigen-specific gamma interferon assays for surveillance of Mycobacterium tuberculosis infections. Clin Vaccine Immunol 2008;15:327-37.

[24] Weinberg A, Song LY, Wilkening C, Sevin A, Blais B, Louzao R, et al Optimization and limitations of use of cryopreserved peripheral blood mononuclear cells for functional and phenotypic T-cell characterization. Clin Vaccine Immunol 2009;16:1176-86. 
[25] Ausiello CM, Urbani F, la Sala A, Lande R, Cassone A. Vaccine- and antigendependent type 1 and type 2 cytokine induction after primary vaccination of infants with whole-cell or acellular pertussis vaccines. Infect Immun 1997;65:2168-74.

[26] Ausiello CM, Lande R, Urbani F, la Sala A, Stefanelli P, Salmaso S, et al. Cell-mediated immune responses in four-year-old children after primary immunization with acellular pertussis vaccines. Infect Immun 1999;67:4064-71.

[27] Ausiello CM, Lande R, Urbani F, Di Carlo B, Stefanelli P, Salmaso S, et al. Cellmediated immunity and antibody responses to Bordetella pertussis antigens in children with a history of pertussis infection and in recipients of an acellular pertussis vaccine. J Infect Dis 2000;181:1989-95.

[28] He Q Tran Minh NN, Edelman K, Viljanen MK, Arvilommi H, Mertsola J. Cytokine mRNA expression and proliferative responses induced by pertussis toxin, filamentous hemagglutinin, and pertactin of Bordetella pertussis in the peripheral blood mononuclear cells of infected and immunized schoolchildren and adults. Infect Immun 1998;66:3796-801.

[29] Mascart F, Verscheure V, Malfroot A, Hainaut M, Piérard D, Temerman S, et al. Bordetella pertussis infection in 2-month-old infants promotes type $1 \mathrm{~T}$ cell responses. J Immunol 2003;170:1504-9.

[30] Silva LC, Ortigosa LC, Benard G. Anti-TNF- $\alpha$ agents in the treatment of immune-mediated inflammatory diseases: mechanisms of action and pitfalls. Immunotherapy 2010;2:817-33.

[31] Reimann KA, Chernoff M, Wilkening CL, Nickerson CE, Landay AL. Preservation of lymphocyte immunophenotype and proliferative responses in cryopreserved peripheral blood mononuclear cells from human immunodeficiency virus type 1-infected donors: implications for multicenter clinical trials, The ACTG Immunology Advanced Technology Laboratories. Clin Diagn Lab Immunol 2000;7:352-9.

[32] Angulo R, Fulcher D. Measurement of Candida-specific blastogenesis: comparison of carboxyfluorescein succinimidyl ester labelling of T cells, thymidine incorporation, and CD69 expression. Cytometry 1998;34:143-51.
[33] Popma SH, Krasinskas AM, McLean AD, Szeto WY, Kreisel D, Moore JS, et al. Immune monitoring in xenotransplantation: the multiparameter flow cytometric mixed lymphocyte culture assay. Cytometry 2000;42:277-83.

[34] Fulcher D, Wong S. Carboxyfluorescein succinimidyl ester-based proliferative assays for assessment of $\mathrm{T}$ cell function in the diagnostic laboratory. Immunol Cell Biol 1999;77:559-64.

[35] Owen RE, Sinclair E, Emu B, Heitman JW, Hirschkorn DF, Epling CL, et al. Loss of $\mathrm{T}$ cell responses following long-term cryopreservation. J Immunol Methods 2007;326:93-115.

[36] Rota MC, Ausiello CM, D’Amelio R, Cassone A, Giammanco A, Molica C, et al. Prevalence of markers of exposure to Bordetella pertussis among Italian young adults. Clin Infect Dis 1998;26:297-302.

[37] Rota MC, D’Ancona F, Massari M, Mandolini D, Giammanco A, Carbonari P, et al How increased pertussis vaccination coverage is changing the epidemiology of pertussis in Italy. Vaccine 2005;23:5299-305.

[38] Venken K, Thewissen M, Hellings N, Somers V, Hensen K, Rummens JL, et al. A CFSE based assay for measuring CD4+CD25+ regulatory T cell mediated suppression of auto-antigen specific and polyclonal T cell responses. J Immunol Methods 2007;322:1-11.

[39] Peters JH, Preijers FW, Woestenenk R, Hilbrands LB, Koenen HJ, Joosten I. Clinical grade Treg: GMP isolation, improvement of purity by CD127 depletion, Treg expansion, and Treg cryopreservation. PLoS One 2008;3:e3161.

[40] Elkord E. Frequency of human T regulatory cells in peripheral blood is significantly reduced by cryopreservation. J Immunol Methods 2009;347:87-90.

[41] Seale AC, de Jong BC, Zaidi I, Duvall M, Whittle H, Rowland-Jones S, et al. Effects of cryopreservation on CD4+ CD25+ T cells of HIV-1 infected individuals. J Clin Lab Anal 2008;22:153-8.

[42] Violette Dirix, Virginie Verscheure, Françoise Vermeulen, Iris De Schutter, Tessa Goetghebuer, Camille Locht, Françoise Mascart. Both CD4+ and CD8+ lymphocytes participate in the IFN- response to filamentous hemagglutinin from Bordetella pertussis in infants, children and adults. Clinical and Developmental Immunology, (in press). 